\title{
EQUIVALENCE AMONG SOME NEIGHBORHOOD STRUCTURES IN FUZZY TOPOLOGY
}

\author{
COSIMO DE MITRI
}

Received 15 March 2005 and in revised form 26 October 2005

We deepen the study of two known neighborhood structures, which here will be called $f \cdot k$-neighborhood structures and $f \cdot q$-neighborhood structures, in the context of Šstak fuzzy topological spaces. In particular, we characterize fuzzy topologies by $f \cdot k$-neighborhood structures. Moreover we introduce and discuss the notions of $f \cdot k$ neighborhood prestructure and $f \cdot m$-neighborhood structure in the same context. At last we prove that the three neighborhood structures mentioned above are equivalent.

\section{Introduction}

The problem of characterizing fuzzy topologies by means of suitable local structures has been investigated by many authors since the end of the seventies. The most important notions of neighborhood considered are essentially three, as many as the principal notions of membership of a fuzzy point to a fuzzy set, which here will be called belonging, proper belonging, and quasi coincidence. For the corresponding notions of neighborhood, we will use, respectively, the terms $k$-neighborhood, m-neighborhood, and $q$ neighborhood.

The $k$-neighborhoods in Chang topological spaces were introduced in 1978 by Kerre [6]. Two years later, in [9], Pu and Liu asserted in a theorem that a certain set of properties of the $k$-neighborhoods characterizes the topology, but afterwards this part of the proposition was shown to be false. The purpose of characterizing Chang topologies through the $k$-neighborhoods was realized in 1983 by De Mitri and Pascali [1] (this result published in [1] is already present in C. De Mitri's Degree thesis, E. Pascali as Supervising Professor, of 1977); in this work, the notions of belonging and proper belonging were used contextually, and this choice made possible the reaching of the expected result.

The characterization of Chang topologies through the $m$-neighborhoods was established in 1981 by de Prada Vicente [2], and soon after by Ghanim and Mashhour [5].

As for the $q$-neighborhoods, they were introduced in [9], already mentioned, and, in this same paper, a set of properties characterizing the topology was listed.

A comparative analysis of the three notions of neighborhood mentioned above was done in 1987 by Kerre and Ottoy [7], and two years later it was taken up again in [8] 
by the same authors. But unfortunately, these two works did not take into account the results contained in [1], so the first of them ends with inexact valuations that have not been denied in the second one, where the authors have stated how it is possible to express any one of the three neighborhood systems in terms of either of the other two.

Finally in 1990, Šostak [11] generalized the notions of $k$-neighborhood and of $q$-neighborhood setting them in the context of Šostak fuzzy topological spaces: as a consequence of the fuzzification of the notion of openness, the fuzzification of the notion of neighborliness was obtained. But also here the results established in [1] have been ignored, and the treatment concerning the $k$-neighborhood system has remained incomplete. We can repeat analogous remarks with regard to the results achieved in 1997 by Demirci [3]. The results established in [11] about the $q$-neighborhoods were found again in 2004 by Fang [4].

This short survey here made about the problem of the neighborhoods is limited to those notions of neighborhood which are analyzed in this paper, and which present a strong homogeneity one another. We left out, for instance, all neighborhood systems relating to crisp points only, for example those of Ludescher, Roventa, and Warren (see [7]), which are set in Chang topological spaces, and those of Ying [12], which are set in fuzzifying topological spaces. Likewise, we left out the neighborhoods of fuzzy points introduced again by Ying in [13]: concerning this, we observe that although the context is that of Chang topological spaces, where the property of openness is crisp, nevertheless these neighborhoods are fuzzified through a fuzzification of the membership relation.

The topic treated in this paper is set in the context of Šostak fuzzy topological spaces [10]. Differently from $[3,11]$, but similarly to [4], the neighborhood structures are introduced in a general form, that is, as functions defined even without a topological structure. In Section 3, dealing with the $k$-neighborhood structure, the notion of fuzzy pretopology is involved too, and so the results contained in [11] are used as a basis for further researches; indeed, in such a context, the opportunity emerges to introduce the notion of $k$-neighborhood prestructure, and we obtain a description of the smallest fuzzy topology among those which are greater than a given fuzzy pretopology. Successively, in the same section, the $k$-neighborhood structures are again seen in the more particular context of Chang spaces, so permitting the improvement of some results achieved in [7] and the comparison with the neighborhood structure studied in [1]. Finally, in the last two sections, the structures of the $m$-neighborhoods and of the $q$-neighborhoods are analyzed, and their link with the $k$-neighborhood structures is established, so proving their essential equivalence.

\section{Preliminaries}

For any nonempty set $X$, we call fuzzy sets of $X$ the elements of $\mathbf{I}^{X}$, where $\mathbf{I}=[0,1]$. Among them, there are in particular the elements of $2^{X}$, where $2=\{0,1\}$, so-named crisp sets; two notable examples are the characteristic functions $\chi_{\varnothing}$ and $\chi_{x}$, which in the sequel we will denote, respectively, by the symbols $\varnothing$ and $X$. If $A \in \mathbf{I}^{X}$, we call the support of $A$ the set $A^{\perp}=\{x \in X \mid A(x)>0\}$.

We call each fuzzy set of $X$ having a singleton as its support a fuzzy point of $X$. If $\{x\}$ is the support of the fuzzy point $p$ and $p(x)=\lambda$, we write $p=x_{\lambda}$. The family of the fuzzy 
points of $X$ will be denoted by $\mathscr{S}(X)$; moreover we put $\mathscr{S}^{*}(X)=\left\{x_{\lambda} \in \mathscr{S}(X) \mid \lambda<1\right\}$. If $p=x_{\lambda} \in \mathscr{S}^{*}(X)$, we call the dual of $p$ the fuzzy point $p^{\prime}=x_{1-\lambda}$.

Given $p=x_{\lambda} \in \mathscr{S}(X)$ and $A \in \mathbf{I}^{X}$, we define the relations of belonging, proper belonging, and quasi coincidence, respectively, as follows: $p \preceq A$ if $\lambda \leq A(x), p \prec A$ if $\lambda<A(x)$, and $p \hat{\mathrm{q}} A$ if $\lambda>1-A(x)$.

For any $\tau \in \mathbf{I}^{\mathbf{I}^{X}}$, we consider the following axioms:

$\left(\mathrm{t}_{1}\right) \tau(\varnothing)=\tau(X)=1$;

$\left(\mathrm{t}_{2}\right)$ for all $A_{1}, A_{2} \in \mathbf{I}^{X}, \tau\left(A_{1} \wedge A_{2}\right) \geq \tau\left(A_{1}\right) \wedge \tau\left(A_{2}\right)$;

$\left(\mathrm{t}_{3}\right)$ for all $\mathscr{A} \subseteq \mathbf{I}^{X}, \tau(\bigvee \mathscr{A}) \geq \bigwedge_{A \in \mathscr{A}} \tau(A)$.

Each map $\tau$ verifying the conditions $\left(\mathrm{t}_{1,2}\right)$ will be called here an $f$-pretopology on $X$; if $\tau$ also verifies the condition $\left(t_{3}\right)$, we will say that $\tau$ is an $f$-topology on $X$. The pair $(X, \tau)$ will be called, respectively, an $f$-pretopological space and an $f$-topological space. If the map $\tau$ is such that $\tau\left(\mathbf{I}^{X}\right) \subseteq 2$, then we will prefer, respectively, the terms $c$-pretopology, c-topology, $c$-pretopological space, and c-topological space; we note that, in this case, $\tau$ is the characteristic function of a subset of $\mathbf{I}^{X}$, which we will indicate by the same symbol $\tau$.

If $\tau$ is a $c$-topology on $X$, given $\beta \subseteq \tau$, we say that $\beta$ is a base of $\tau$ if for all $A \in \tau$, there exists $\beta^{\prime} \subseteq \beta$ such that $A=\bigvee \beta^{\prime}$.

We have that if $\delta$ is a $c$-pretopology, then the family $\tau=\left\{A \in \mathbf{I}^{X} \mid \exists \delta^{\prime} \subseteq \delta\right.$ such that $\left.A=\bigvee \delta^{\prime}\right\}$ is the only $c$-topology of which $\delta$ is a base, and it is the coarsest $c$-topology among the ones which contain $\delta$; or rather we observe that $\tau$ is the smallest $f$-topology among the ones which are greater than $\delta$.

Given the $f$-topological spaces $(X, \tau)$ and $(Y, \delta)$, any function $f: X \rightarrow Y$ is said to be continuous if for all $B \in \delta, \tau\left(f^{-}(B)\right) \geq \delta(B)$, where $f^{-}(B)=B \circ f$. Moreover, we recall that for any $x_{\lambda} \in \mathscr{S}(X), f \rightarrow\left(x_{\lambda}\right)=(f(x))_{\lambda} \in \mathscr{S}(Y)$.

\section{3. $k$-neighborhood structures and prestructures}

For any $\kappa \in \mathbf{I}^{\varphi(X) \times \mathbf{I}^{X}}$, where $X$ is a nonempty set, we can consider the following axioms:

$\left(\mathrm{k}_{0}\right)$ for all $p \in \mathscr{S}(X), \bigvee_{U \in \mathbf{I}^{X}} \kappa(p, U)=1$;

$\left(\mathrm{k}_{1}\right)$ for all $p \in \mathscr{S}(X)$ and for all $U, V \in \mathbf{I}^{X}$, if $U \leq V$, then $\kappa(p, U) \leq \kappa(p, V)$;

$\left(\mathrm{k}_{2}\right)$ for all $p \in \mathscr{S}(X)$ and for all $U_{1}, U_{2} \in \mathbf{I}^{X}, \kappa\left(p, U_{1} \wedge U_{2}\right) \geq \kappa\left(p, U_{1}\right) \wedge \kappa\left(p, U_{2}\right)$;

$\left(\mathrm{k}_{3}\right)$ for all $p \in \mathscr{S}(X)$ and for all $U \in \mathbf{I}^{X}$, if $\kappa(p, U)>0$, then $p \preceq U$;

$\left(\mathrm{k}_{4}\right)$ for all $p \in \mathscr{S}(X)$ and for all $U \in \mathbf{I}^{X}, \kappa(p, U) \leq \bigvee_{V \leq U}\left(\kappa(p, V) \wedge\left(\bigwedge_{q \leq V} \kappa(q, V)\right)\right)$;

$\left(\mathrm{k}_{5}\right)$ for all $p \in \mathscr{S}(X)$ and for all $U \in \mathbf{I}^{X}, \kappa(p, U) \geq \bigwedge_{q<p} \kappa(q, U)$.

We observe that the second member of the inequality in $\left(\mathrm{k}_{4}\right)$, in the presence of the condition $\left(\mathrm{k}_{3}\right)$, shrinks to $\bigvee_{p \leq V \leq U} \bigwedge_{q \leq V} \kappa(q, V)$.

The axioms $\left(\mathrm{k}_{0,1,2,3,4}\right)$ are taken from $[3,11]$; however the axiom $\left(\mathrm{k}_{5}\right)$ is new, although deduced from [1], and we prove (see Example 3.10) that it is independent of the others. We note that the axioms $\left(k_{3,4}\right)$ involve the relation of belonging, while the axiom $\left(k_{5}\right)$ involves the relation of proper belonging.

Every map $\kappa \in \mathbf{I}^{\mathscr{S}(X) \times \mathbf{I}^{X}}$ verifying the conditions $\left(\mathrm{k}_{0,1,2,3,4}\right)$ will be called an $f \cdot k$ neighborhood prestructure, or an $f \cdot k$-prestructure, on $X$; if $\kappa$ also verifies the condition $\left(\mathrm{k}_{5}\right)$, we will say that $\kappa$ is an $f \cdot k$-neighborhood structure, or an $f \cdot k$-structure, on $X$. 
3912 Equivalence among some fuzzy neighborhood structures

Proposition 3.1. Let $\kappa \in \mathbf{I}^{\varphi(X) \times \mathbf{I}^{X}}$.

If $\kappa$ satisfies the conditions $\left(\mathrm{k}_{0,1}\right)$, then it also satisfies the condition

$\left(\mathrm{k}_{\mathrm{a}}\right)$ for all $p \in \mathscr{S}(X), \kappa(p, X)=1$.

If $\kappa$ satisfies the conditions $\left(\mathrm{k}_{1,3,4}\right)$, then it also satisfies the condition

$\left(\mathrm{k}_{\mathrm{b}}\right)$ for all $p, q \in \mathscr{S}(X)$ and for all $U \in \mathbf{I}^{X}$, if $q \prec p$, then $\kappa(p, U) \leq \kappa(q, U)$.

If $\kappa$ satisfies the condition $\left(\mathrm{k}_{5}\right)$, then it also satisfies the condition

$\left(\mathrm{k}_{\mathrm{c}}\right)$ for all $U \in \mathbf{I}^{X}, \bigwedge_{p<U} \kappa(p, U) \leq \bigwedge_{p \leq U} \kappa(p, U)$.

If $\kappa$ satisfies the conditions $\left(\mathrm{k}_{1,3,4,5}\right)$, then it also satisfies the condition

$\left(\mathrm{k}_{\mathrm{k}}\right)$ for all $p \in \mathscr{S}(X)$ and for all $U \in \mathbf{I}^{X}, \kappa(p, U)=\bigwedge_{q<p} \kappa(q, U)$.

Proof. The check of $\left(\mathrm{k}_{\mathrm{a}}\right)$ under the conditions $\left(\mathrm{k}_{0,1}\right)$ is immediate.

In order to prove $\left(\mathrm{k}_{\mathrm{b}}\right)$ under the hypothesies $\left(\mathrm{k}_{1,3,4}\right)$, we suppose on the contrary that $p, q \in \mathscr{S}(X)$ exist such that $q \prec p$ and $\kappa(q, U)<\kappa(p, U)$. Then, by $\left(\mathrm{k}_{3,4}\right), \kappa(q, U)<$ $\bigvee_{p \leq V \leq U} \bigwedge_{r \leq V} \kappa(r, V)$, hence $V \in \mathbf{I}^{X}$ exists such that $p \preceq V \leq U$ and $\kappa(q, U)<\bigwedge_{r \leq V}$ $\kappa(r, V)$. From this it follows, since $q \preceq V$, that $\kappa(q, U)<\kappa(q, V)$, in contrast with $\left(\mathrm{k}_{1}\right)$.

As for $\left(\mathrm{k}_{\mathrm{c}}\right)$ under the condition $\left(\mathrm{k}_{5}\right)$, we only need to observe that for all $p \preceq U$, it holds that $\bigwedge_{q<U} \kappa(q, U) \leq \bigwedge_{q<p} \kappa(q, U) \leq \kappa(p, U)$.

Finally, we recognize that the condition $\left(k_{k}\right)$ trivially follows from the conditions $\left(k_{5}\right)$ and $\left(\mathrm{k}_{\mathrm{b}}\right)$.

With any map $\tau \in \mathbf{I}^{\mathbf{I}^{X}}$, we associate the map $\mathscr{K}_{\tau} \in \mathbf{I}^{\mathscr{\varphi}(X) \times \mathbf{I}^{X}}$ defined by

$$
\forall p \in \mathscr{S}(X), \forall U \in \mathbf{I}^{X}, \quad \mathscr{K}_{\tau}(p, U)=\bigvee_{p \leq A \leq U} \tau(A)
$$

On the other hand, with any map $\kappa \in \mathbf{I}^{\mathscr{G}(X) \times \mathbf{I}^{X}}$, one can associate the maps $\mathscr{D}_{\kappa}, \mathscr{T}_{\kappa} \in \mathbf{I}^{\mathbf{I}^{X}}$ defined by

$$
\forall A \in \mathbf{I}^{X}, \quad \mathscr{D}_{\kappa}(A)=\bigwedge_{p \succeq A} \kappa(p, A), \quad \mathscr{T}_{\kappa}(A)=\bigwedge_{p<A} \kappa(p, A)
$$

It is immediate to note that $\mathscr{D}_{\kappa} \leq \mathscr{T}_{\kappa}$ in general, and that $\mathscr{D}_{\kappa}=\mathscr{T}_{\kappa}$ if $\kappa$ satisfies the condition $\left(\mathrm{k}_{\mathrm{c}}\right)$.

Now we present an example of an $f \cdot k$-structure. The case of an $f \cdot k$-prestructure which is not an $f \cdot k$-structure is showed forward (see Example 3.10).

Example 3.2. Let $X$ be a singleton $\{x\}$. For $\lambda \in(0,1]$ and $U \in \mathbf{I}^{X}$, we define

$$
\kappa\left(x_{\lambda}, U\right)= \begin{cases}U(x) & \text { if } \lambda \leq U(x), \\ 0 & \text { if } \lambda>U(x) .\end{cases}
$$

It is easy to check that $\kappa$ satisfies the conditions $\left(\mathrm{k}_{0,1,2,3,4,5}\right)$.

Moreover, we have

$$
\forall A \in \mathbf{I}^{X}, \quad \mathscr{D}_{\kappa}(A)=\mathscr{T}_{\kappa}(A)= \begin{cases}A(x) & \text { if } A(x)>0 \\ 1 & \text { if } A(x)=0 .\end{cases}
$$


The following theorem includes [3, Proposition 2.5] and [11, Theorems 1 and 3], and furthermore it states that if $\tau$ is an $f$-topology on $X$, then $\mathcal{K}_{\tau}$ is an $f \cdot k$-structure on $X$.

Theorem 3.3. If $\tau$ is an $f$-pretopology on $X$, then $\mathcal{K}_{\tau}$ is an $f \cdot k$-prestructure on $X$, and $\tau \leq \mathscr{D}_{K_{\tau}}$.

If $\tau$ is an $f$-topology on $X$, then $\mathscr{K}_{\tau}$ is an $f \cdot k$-structure on $X$, and $\tau=\mathscr{D}_{\mathscr{K}_{\tau}}=\mathscr{T}_{\mathscr{K}_{\tau}}$.

Proof. As for the proof of the first part and of the equality $\tau=\mathscr{D}_{\mathscr{K}_{\tau}}$ at the end of the second part, see [3, Proposition 2.5] and [11, Theorems 1 and 3].

Now we prove that if $\tau$ is an $f$-topology, then $\mathscr{K}_{\tau}$ also satisfies the condition $\left(\mathrm{k}_{5}\right)$. Given $p \in \mathscr{S}(X), U \in \mathbf{I}^{X}$, and $\alpha>\mathscr{K}_{\tau}(p, U)$, we claim that there exists $q \prec p$ such that $\mathscr{K}_{\tau}(q, U) \leq \alpha$. Instead, we suppose that for all $q \prec p \mathscr{K}_{\tau}(q, U)>\alpha$, that is, $A_{q} \in \mathbf{I}^{X}$ exists such that $q \preceq A_{q} \leq U$ and $\tau\left(A_{q}\right)>\alpha$. If $A=\bigvee_{q<p} A_{q}$, we have that $p=\bigvee_{q<p} q \preceq A \leq U$. Moreover, by $\left(\mathrm{t}_{3}\right)$, one has that $\tau(A) \geq \bigwedge_{q<p} \tau\left(A_{q}\right) \geq \alpha$, hence $\tau(A)>\mathscr{K}_{\tau}(p, U)$, which obviously contradicts the definition of $\mathscr{K}_{\tau}(p, U)$.

Finally, we observe that by the condition $\left(\mathrm{k}_{\mathrm{c}}\right)$ applied to $\mathscr{K}_{\tau}$, it follows that $\mathscr{D}_{\mathscr{K}_{\tau}}=$ $\mathscr{T}_{\mathscr{K}_{\tau}}$.

Remark 3.4. If $\tau$ is an $f$-topology on $X$, then by the theorem above, we have $\tau(A)=$ $\bigwedge_{p \leq A} \mathcal{K}_{\tau}(p, A)$ for all $A \in \mathbf{I}^{X}$ (we note more precisely, due to the condition $\left(\mathrm{k}_{\mathrm{b}}\right)$ applied to $\mathcal{K}_{\tau}$, that $\left.\tau(A)=\bigwedge_{x \in A^{\perp}} \mathscr{K}_{\tau}\left(x_{A(x)}, A\right)\right)$. Thus the $f$-topology $\tau$ can be expressed in terms of the $f \cdot k$-structure associated with it.

Moreover, since also the equality $\tau(A)=\bigwedge_{p \prec A} \mathcal{K}_{\tau}(p, A)$, for all $A \in \mathbf{I}^{X}$, holds, one can assert that although the function $\mathscr{K}_{\tau}$ is defined on $\mathscr{S}(X) \times \mathbf{I}^{X}$, its restriction to $\mathscr{S}^{*}(X) \times \mathbf{I}^{X}$ is enough to characterize the $f$-topology $\tau$.

The following two theorems concern both the maps $\mathscr{D}_{\kappa}$ and $\mathscr{T}_{\kappa}$ generated by a map $\kappa \in \mathbf{I}^{\mathscr{\varphi}(X) \times \mathbf{I}^{X}}$; the statements relating only to $\mathscr{D}_{\kappa}$ appear in [11, Theorem 2], while the ones involving also $\mathscr{T}_{\kappa}$ are new.

Theorem 3.5. If $\kappa$ is an $f \cdot k$-prestructure on $X$, then $\mathscr{D}_{\kappa}$ is an f-pretopology on $X$, and $\kappa=\mathscr{K}_{\mathscr{D}_{\kappa}}$. Moreover $\mathscr{T}_{\kappa}$ is an f-topology on $X$, and $\kappa \leq \mathscr{K}_{\mathscr{T}_{\kappa}}$.

Proof. As for the proof of the first part, see [11, Theorem 2].

Now we prove that $\mathscr{T}_{\kappa}$ is an $f$-topology on $X$. The check of the conditions $\left(\mathrm{t}_{1,2}\right)$ can be done in the same way as for $\mathscr{D}_{\mathcal{K}}$ in [11]. There remains to check the condition $\left(\mathrm{t}_{3}\right)$ : given $\mathscr{A} \subseteq \mathbf{I}^{X}$, we claim that $\mathscr{T}_{\kappa}(\bigvee \mathscr{A}) \geq \bigwedge_{A \in \mathscr{A}} \mathscr{T}_{\kappa}(A)$, that is, $\bigwedge_{p \prec \bigvee \mathscr{A}} \kappa(p, \bigvee \mathscr{A}) \geq \bigwedge_{A \in \mathscr{A}} \mathscr{T}_{\kappa}(A)$ In fact, given $p \prec \vee \mathscr{A}$ and $A \in \mathscr{A}$ such that $p \prec A$, we have, using the condition $\left(\mathrm{k}_{1}\right)$, that $\kappa(p, \bigvee \mathscr{A}) \geq \kappa(p, A) \geq \mathscr{T}_{\kappa}(A) \geq \bigwedge_{A \in \mathscr{A}} \mathscr{T}_{\kappa}(A)$

Finally, we observe that the relation $\kappa \leq \mathscr{K}_{\mathscr{T}_{\kappa}}$ trivially follows from the already proved fact that $\kappa=\mathscr{K}_{\mathscr{D}_{\kappa}}$ and from the fact that since $\mathscr{D}_{\mathcal{K}} \leq \mathscr{T}_{\kappa}$, it obviously follows that $\mathscr{K}_{\mathscr{D}_{\kappa}} \leq$ $\mathcal{K}_{\mathscr{T}_{\kappa}}$.

Theorem 3.6. If $\kappa$ is an $f \cdot k$-structure on $X$, then $\mathscr{T}_{\kappa}$ is an $f$-topology on $X$, and the equalities $\mathscr{T}_{\kappa}=\mathscr{D}_{\kappa}$ and $\kappa=\mathscr{K}_{\mathcal{T}_{\kappa}}$ hold.

Proof. By the condition $\left(\mathrm{k}_{\mathrm{c}}\right)$, a consequence of $\left(\mathrm{k}_{5}\right)$, we have that $\mathscr{T}_{\kappa}=\mathscr{D}_{\kappa}$. So the thesis trivially follows from Theorem 3.5. 
We note that by Theorems 3.3 and 3.6, if $\tau$ is an $f$-topology on $X$, then $\mathscr{K}_{\tau}$ is the only $f \cdot k$-structure on $X$ such that $\tau=\mathscr{T}_{\mathscr{K}_{\tau}}$; similarly, if $\kappa$ is an $f \cdot k$-structure on $X$, then $\mathscr{T}_{\kappa}$ is the only $f$-topology on $X$ such that $\kappa=\mathscr{K}_{\mathcal{F}_{\kappa}}$.

Remark 3.7. From Theorems 3.3 and 3.5, we easily deduce that if $\kappa$ is an $f \cdot k$-prestructure without being an $f \cdot k$-structure, then the $f$-pretopology $\mathscr{D}_{\kappa}$ cannot be an $f$-topology, and furthermore the equality $\kappa=\mathscr{K}_{\mathscr{T}_{\kappa}}$ cannot hold.

On the other hand, it is proved (see Example 3.12) that some $f$-pretopology $\tau$ exists which is not an $f$-topology, and nevertheless it is such that $\mathcal{K}_{\tau}$ is an $f \cdot k$-structure. For such an $f$-pretopology, the equality $\tau=\mathscr{D}_{\mathscr{K}_{\tau}}$ cannot be true, since, due to Theorem 3.6, $D_{\mathscr{K}_{\tau}}$ is an $f$-topology. Consequently, by Theorem 3.5, we can exclude the existence of an $f \cdot k$-prestructure $\kappa$ such that $\mathscr{D}_{\mathcal{K}}=\tau$, since otherwise we would have $\mathscr{K}_{\tau}=\mathscr{K}_{\mathscr{D}_{\kappa}}=\kappa$ and hence $\tau=\mathscr{D}_{\mathscr{K}_{\tau}}$.

Theorems 3.3 and 3.5 suggest a procedure to build $f \cdot k$-structures from $f \cdot k$-prestructures and $f$-topologies from $f$-pretopologies: in fact, with the $f \cdot k$-prestructure $\kappa$, one can associate the $f \cdot k$-structure $\mathscr{K}_{\mathcal{F}_{\kappa}}$ and with the $f$-pretopology $\tau$, one can associate the $f$-topology $\mathscr{T}_{\mathscr{K}_{\tau}}$.

We know that the relations $\kappa \leq \mathscr{K}_{\mathscr{T}_{\kappa}}$ and $\tau \leq \mathscr{T}_{\mathscr{K}_{\tau}}$ hold; the following theorem states that $\mathscr{Y}_{\mathscr{T}_{\kappa}}$ is the smallest $f \cdot k$-structure among the ones which are greater than $\kappa$, and that $\mathscr{T}_{\mathscr{K}_{\tau}}$ is the smallest $f$-topology among the ones which are greater than $\tau$.

THeOREm 3.8. If $\kappa$ is an $f \cdot k$-prestructure on $X$, then $\mathscr{Y}_{\mathscr{T}_{\kappa}}$ is the smallest $f \cdot k$-structure on $X$ such that $\kappa \leq \mathscr{K}_{\mathscr{T}_{\kappa}}$.

If $\tau$ is an $f$-pretopology on $X$, then $\mathscr{T}_{\mathscr{K}_{\tau}}$ is the smallest f-topology on $X$ such that $\tau \leq \mathscr{T}_{\mathscr{K}_{\tau}}$.

Proof. Let $\kappa^{\prime}$ be an $f \cdot k$-structure such that $\kappa^{\prime} \geq \kappa$, and take $p \in \mathscr{S}(X)$ and $U \in \mathbf{I}^{X}$. By applying $\left(\mathrm{k}_{1}\right)$ to $\kappa$ and $\left(\mathrm{k}_{5}\right)$ to $\kappa^{\prime}$, one gets that for all $A \in \mathbf{I}^{X}$, if $p \preceq A \leq U$, then $\mathscr{T}_{\kappa}(A)=\bigwedge_{q<A} \kappa(q, A) \leq \bigwedge_{q<A} \kappa(q, U) \leq \bigwedge_{q<p} \kappa(q, U) \leq \bigwedge_{q<p} \kappa^{\prime}(q, U) \leq \kappa^{\prime}(p, U)$. Hence, $\mathcal{K}_{\mathscr{T}_{\kappa}}(p, U)=\bigwedge_{q<A} \kappa(q, A) \leq \kappa^{\prime}(p, U)$.

Now, let $\tau^{\prime}$ be an $f$-topology such that $\tau^{\prime} \geq \tau$, and assume on the contrary that there exists $A \in \mathbf{I}^{X}$ such that $\tau^{\prime}(A)<\mathscr{T}_{\mathscr{K}_{\tau}}(A)$. Taking $\alpha \in\left(\tau^{\prime}(A), \mathscr{T}_{\mathscr{K}_{\tau}}(A)\right)$, for all $p \prec A$, since $\alpha<\bigvee_{p \leq U \leq A} \tau(U)$, there would exist $A_{p} \in \mathbf{I}^{X}$ such that $p \preceq A_{p} \leq A$ and $\alpha<\tau\left(A_{p}\right)$. Since $A=\bigvee_{p \prec A} A_{p}$, using the condition $\left(\mathrm{t}_{3}\right)$ we would have $\tau^{\prime}(A) \geq \bigwedge_{p \prec A} \tau^{\prime}\left(A_{p}\right) \geq \bigwedge_{p \prec A} \tau\left(A_{p}\right)$, and hence $\tau^{\prime}(A) \geq \alpha$.

The theorem below characterizes the continuity of the functions between $f$-topological spaces by means of the related $f \cdot k$-structures.

Theorem 3.9. Let $(X, \tau)$ and $(Y, \delta)$ be two f-topological spaces. The map $f: X \rightarrow Y$ is continuous if and only if, for all $p \in \mathscr{S}(X)$ and for all $V \in \mathbf{I}^{Y}, \mathscr{K}_{\tau}\left(p, f^{-}(V)\right) \geq \mathscr{K}_{\delta}\left(f^{-}(p), V\right)$.

Proof. The proof is straightforward.

If the map $\kappa \in \mathbf{I}^{\mathscr{\varphi}(X) \times \mathbf{I}^{X}}$ is such that $\kappa\left(\mathscr{S}(X) \times \mathbf{I}^{X}\right) \subseteq 2$, we use the terms $c \cdot k$ neighborhood structure and prestructure, or shortly $c \cdot k$-structure and prestructure. If we define that for all $p \in \mathscr{S}(X), \kappa\langle p\rangle=\left\{U \in \mathbf{I}^{X} \mid \kappa(p, U)=1\right\}$, then the axioms $\left(\mathrm{k}_{0,1,2,3,4,5}\right)$ 
can be written, respectively, as follows:

$\left(\mathrm{k}_{0}^{c}\right)$ for all $p \in \mathscr{S}(X), \kappa\langle p\rangle \neq \varnothing$;

$\left(\mathrm{k}_{1}^{c}\right)$ for all $p \in \mathscr{S}(X)$ and for all $U, V \in \mathbf{I}^{X}$, if $U \in \kappa\langle p\rangle$ and $U \leq V$, then $V \in \kappa\langle p\rangle$;

$\left(\mathrm{k}_{2}^{c}\right)$ for all $p \in \mathscr{S}(X)$ and for all $U_{1}, U_{2} \in \mathbf{I}^{X}$, if $U_{1}, U_{2} \in \kappa\langle p\rangle$, then $U_{1} \wedge U_{2} \in \kappa\langle p\rangle$;

$\left(\mathrm{k}_{3}^{c}\right)$ for all $p \in \mathscr{S}(X)$ and for all $U \in \mathbf{I}^{X}$, if $U \in \kappa\langle p\rangle$, then $p \preceq U$;

$\left(\mathrm{k}_{4}^{c}\right)$ for all $p \in \mathscr{S}(X)$ and for all $U \in \mathbf{I}^{X}$, if $U \in \kappa\langle p\rangle$, then there exist $V \in \kappa\langle p\rangle$ such that $V \leq U$ and $V \in \kappa\langle q\rangle$ for all $q \preceq V$;

$\left(\mathrm{k}_{5}^{c}\right)$ for all $p \in \mathscr{S}(X)$ and for all $U \in \mathbf{I}^{X}$, if $U \in \kappa\langle q\rangle$ for all $q \prec p$, then $U \in \kappa\langle p\rangle$.

Moreover, the conditions $\left(\mathrm{k}_{\mathrm{a}, \mathrm{b}, \mathrm{c}, \mathrm{k}}\right)$ become, respectively, the following:

$\left(\mathrm{k}_{\mathrm{a}}^{c}\right)$ for all $p \in \mathscr{S}(X), X \in \kappa\langle p\rangle$;

$\left(\mathrm{k}_{\mathrm{b}}^{c}\right)$ for all $p, q \in \mathscr{S}(X)$, if $q \prec p$, then $\kappa\langle p\rangle \subseteq \kappa\langle q\rangle$;

$\left(\mathrm{k}_{\mathrm{c}}^{c}\right)$ for all $U \in \mathrm{I}^{X}$, if $U \in \kappa\langle p\rangle$ for all $p \prec U$, then $U \in \kappa\langle p\rangle$ for all $p \preceq U$;

$\left(\mathrm{k}_{\mathrm{k}}^{c}\right)$ for all $p \in \mathscr{S}(X), \kappa\langle p\rangle=\bigcap_{q<p} \kappa\langle q\rangle$.

We observe that the conditions $\left(\mathrm{k}_{0,1,2,3,4}^{c}\right)$ appear, nearly the same (see also Remark 3.11), in $[1,6,9]$, and that $\left(\mathrm{k}_{5}^{c}\right)$ appears in [1]. Moreover we recognize that $\left(\mathrm{k}_{\mathrm{k}}^{c}\right)$ constitutes a particular case of that property which in [8, Proposition 2] is called the "inf-completeness property."

Previously it has been asserted, with respect to any map $\kappa \in \mathbf{I}^{\mathscr{S}(X) \times \mathbf{I}^{X}}$, that the condition $\left(\mathrm{k}_{5}\right)$ cannot be deduced from the ones which precede it. Indeed the following example, drawn from [11], shows that the condition $\left(\mathrm{k}_{5}\right)$ is not a consequence of the conditions $\left(\mathrm{k}_{0,1,2,3,4}\right)$ even in the particular case where $\kappa\left(\mathscr{Y}(X) \times \mathbf{I}^{X}\right) \subseteq \mathbf{2}$.

Example 3.10. We define the map $\kappa \in 2^{\mathscr{\varphi}(X) \times \mathbf{I}^{X}}$ by setting for all $p=x_{\lambda} \in \mathscr{S}(X)$ and for all $U \in \mathbf{I}^{X}, \kappa(p, U)=1 \Leftrightarrow \lambda<1 / 2$ and $U(x) \geq \lambda$ or $\lambda \geq 1 / 2$ and $U(x)=1$. In [11], it is observed that $\kappa$ satisfies the conditions $\left(\mathrm{k}_{0,1,2,3,4}^{c}\right)$. It is easy to check that, however, the condition $\left(\mathrm{k}_{5}^{c}\right)$ is not satisfied. Indeed, given $p=x_{1 / 2} \in \mathscr{Y}(X)$ and $U \in \mathbf{I}^{X}$ such that $1 / 2 \leq$ $U(x)<1$, one has that $U \in \kappa\langle q\rangle$ for all $q \prec p$ and that $U \notin \kappa\langle p\rangle$.

Remark 3.11. In [1], dealing with any map $\kappa \in 2^{\varphi(X) \times \mathbf{I}^{X}}$, the following condition appears, very similar to $\left(\mathrm{k}_{4}^{c}\right)$ :

$\left(\mathrm{k}_{\tilde{4}}^{c}\right)$ for all $p \in \mathscr{S}(X)$ and for all $U \in \mathbf{I}^{X}$, if $U \in \kappa\langle p\rangle$, then there exists $V \in \kappa\langle p\rangle$ such that $U \in \kappa\langle q\rangle$ for all $q \prec V$.

We can prove what follows. If $\kappa$ satisfies the conditions $\left(\mathrm{k}_{1,4}^{c}\right)$, for example, if $\kappa$ is a $c \cdot k$ prestructure, then it also satisfies the condition $\left(k_{\tilde{4}}^{c}\right)$; the conditions $\left(k_{1,3, \tilde{4}, 5}^{c}\right)$ imply the condition $\left(\mathrm{k}_{4}^{c}\right)$, so that in the definition of $c \cdot k$-structure, the condition $\left(\mathrm{k}_{4}^{c}\right)$ can be replaced by the condition $\left(\mathrm{k}_{\tilde{4}}^{c}\right)$; however this change cannot be made in the definition of $c \cdot k$-prestructure, which means that maps $\kappa$ in $2^{\varphi(X) \times I^{X}}$ exist satisfying the conditions $\left(\mathrm{k}_{0,1,2,3, \tilde{4}}^{c}\right)$ but not the condition $\left(\mathrm{k}_{4}^{c}\right)$.

If $\tau \in 2^{\mathrm{I}^{X}}$, then $\mathscr{K}_{\tau} \in 2^{\mathscr{S}(X) \times \mathbf{I}^{X}}$, and for all $p \in \mathscr{S}(X), \mathscr{K}_{\tau}\langle p\rangle=\left\{U \in \mathbf{I}^{X} \mid \exists A \in \tau\right.$ such that $p \preceq A \leq U\}$. If $\tau$ is a $c$-pretopology, then for all $p \in \mathscr{S}(X)$, the elements of $\mathscr{K}_{\tau}\langle p\rangle$ are the $k$-neighborhoods of $p$ with respect to $\tau$.

If $\kappa \in 2^{\mathscr{S}(X) \times \mathbf{I}^{X}}$, then $\mathscr{D}_{\kappa}, \mathscr{T}_{\kappa} \in 2^{\mathbf{I}^{X}}$, and we obtain that $\mathscr{D}_{\kappa}=\left\{A \in \mathbf{I}^{X} \mid A \in \kappa\langle p\rangle \forall p \preceq\right.$ $A\}$ and $\mathscr{T}_{\kappa}=\left\{A \in \mathbf{I}^{X} \mid A \in \kappa\langle p\rangle \forall p \prec A\right\}$, where $\mathscr{D}_{\kappa} \subseteq \mathscr{T}_{\kappa}$ in general and $\mathscr{D}_{\kappa}=\mathscr{T}_{\kappa}$ if $\kappa$ satisfies the condition $\left(\mathrm{k}_{\mathrm{c}}^{c}\right)$. 
From Theorem 3.3, we deduce that if $\tau$ is a $c$-pretopology, then $\mathscr{K}_{\tau}$ is a $c \cdot k$ prestructure and $\tau \subseteq \mathscr{D}_{\mathscr{K}_{\tau}}$. Moreover, also owing to Remark 3.4, if $\tau$ is a $c$-topology, then $\mathscr{K}_{\tau}$ is a $c \cdot k$-structure, and for all $A \in \mathbf{I}^{X}, A \in \tau \Leftrightarrow A \in \mathscr{K}_{\tau}\langle p\rangle$ for all $p \preceq A \Leftrightarrow A \in \mathscr{K}_{\tau}\langle p\rangle$ $\forall p \prec A \Leftrightarrow A \in \mathcal{K}_{\tau}\left\langle x_{A(x)}\right\rangle$ for all $x \in A^{\perp}$.

As a confirmation of what has been announced in the second part of Remark 3.7, the example below shows that, in order that $\mathscr{K}_{\tau}$ be a $k$-structure, it is not necessary for $\tau$ to be a topology (and hence the equality $\tau=\mathscr{D}_{\mathscr{K}_{\tau}}$ is not true in general), even if one only considers Chang spaces.

Example 3.12. Let $X=\mathbb{R}$. With every fuzzy point $p=x_{\lambda} \in \mathscr{Y}(\mathbb{R})$, we associate the fuzzy set $C_{p}$ defined by $C_{p}(t)=\max \{\lambda-|t-x|, 0\}$ for all $t \in \mathbb{R}$, and we take $\tau=\left\{C_{p} \mid p \in\right.$ $\mathscr{S}(\mathbb{R})\} \cup\{\varnothing, \mathbb{R}\}$. We recognize that $\tau$ is a $c$-pretopology which does not satisfy the condition $\left(\mathrm{t}_{3}\right)$. We also see that $\mathcal{K}_{\tau}$ is a $c \cdot k$-structure.

From Theorem 3.5, we deduce that if $\kappa \in 2^{\mathscr{P}(X) \times \mathbf{I}^{X}}$ is a $c \cdot k$-prestructure, then $\mathscr{D}_{\kappa}$ and $\mathscr{T}_{\kappa}$ are, respectively, a $c$-pretopology and a $c$-topology. The following proposition states that $\mathscr{T}_{\kappa}$ is just the $c$-topology of which $\mathscr{D}_{\kappa}$ is a base.

Proposition 3.13. If $\kappa \in 2^{\mathscr{\varphi ( X )} \times \mathbf{I}^{X}}$ is a $c \cdot k$-prestructure, then the c-pretopology $\mathscr{D}_{\kappa}$ is a base of the c-topology $\mathscr{T}_{\kappa}$.

Proof. It is already known that $\mathscr{D}_{\kappa} \subseteq \mathscr{T}_{\kappa}$. Given $A \in \mathscr{T}_{\kappa}$, if $p$ is any fuzzy point such that $p \prec A$, owing to the definition of $\mathscr{T}_{\kappa}$, we have that $A \in \kappa\langle p\rangle$, and hence because of $\left(\mathrm{k}_{4}^{c}\right)$, there exist $V_{p} \in \kappa\langle p\rangle$ such that $V_{p} \leq A$ and $V_{p} \in \kappa\langle q\rangle$ for all $q \preceq V_{p}$. The condition $\left(\mathrm{k}_{3}^{c}\right)$ ensures that $p \preceq V_{p}$, and furthermore it holds that $V_{p} \in \mathscr{D}_{\kappa}$ owing to the definition of $\mathscr{D}_{\kappa}$. Thus, taking $\delta^{\prime}=\left\{V_{p} \in \mathbf{I}^{X} \mid p \in \mathscr{S}(X)\right.$ and $\left.p \prec A\right\}$, one has that $\delta^{\prime} \subseteq \mathscr{D}_{\mathcal{K}}$ and $\bigvee_{p<A} p \leq$ $\bigvee_{p \prec A} V_{p} \leq A$, and hence $A=\bigvee \delta^{\prime}$.

Remark 3.14. In general, given a $c$-pretopology $\delta$ and denoting by $\tau$ the $c$-topology of which $\delta$ is a base, we obviously have that, for all $p \in \mathscr{S}(X), \mathscr{K}_{\delta}\langle p\rangle \subseteq \mathscr{K}_{\tau}\langle p\rangle$. As for the reverse inclusion $\mathscr{K}_{\tau}\langle p\rangle \subseteq \mathscr{K}_{\delta}\langle p\rangle$, we observe that it is not necessarily true. Indeed, if it were true that in all cases $\mathscr{K}_{\delta}\langle p\rangle=\mathscr{K}_{\tau}\langle p\rangle$ for all $p \in \mathscr{Y}(X)$, then from Theorem 3.5 and Proposition 3.13, it would follow that every $c \cdot k$-prestructure $\kappa$ generates a $c$-topology $\mathscr{T}_{\kappa}$ such that $\kappa=\mathscr{K}_{\mathscr{T}_{\kappa}}$, in contrast with what has been asserted in the first part of Remark 3.7.

The fact that the above-mentioned inclusion is not true in general constitutes an element of distinction of fuzzy topology from classic topology, and it is essentially due to the fact that the notion of $k$-neighborhood is based on a belonging relation which weakly interacts with the union operation.

Remark 3.15. From Theorem 3.8, it follows that if $\tau$ is a $c$-pretopology, then $\mathscr{T}_{\mathscr{K}_{\tau}}$ is the coarsest $c$-topology among the ones which contain $\tau$, and hence it is just the $c$-topology of which $\tau$ is a base. If we apply this conclusion to the $c$-pretopology $\mathscr{D}_{\mathcal{K}}$ considered in Proposition 3.13, we can get an indirect proof of the proposition itself, simply by observing that because of Theorem 3.5, it follows that $\mathscr{T}_{\mathscr{K}_{\mathscr{T}_{\kappa}}}=\mathscr{T}_{\kappa}$.

From Theorem 3.6, we deduce that if $\kappa \in 2^{\mathscr{P}(X) \times \mathbf{I}^{X}}$ is a $c \cdot k$-structure, then $\mathscr{T}_{\kappa}\left(=\mathscr{D}_{\kappa}\right)$ is a $c$-topology and that for all $p \in \mathscr{S}(X), \kappa\langle p\rangle=\mathscr{K}_{\mathscr{T}_{\kappa}}\langle p\rangle$. Keeping in mind Remark 3.11, we 
can assert that this result is equivalent to [1, Theorem 2.1]. Clearly, the $k$-neighborhood structure gives a suitable extension of the neighborhood structure of classic topology, as well as invalidating the final comment of [7], where the $k$-neighborhood structure was essentially excluded from consideration.

\section{4. $m$-neighborhood structures}

We call every map $\mu \in \mathbf{I}^{\varphi *(X) \times \mathbf{I}^{X}}$ verifying the following axioms an $f \cdot m$-neighborhood structure, or an $f \cdot m$-structure, on the nonempty set $X$ :

$\left(\mathrm{m}_{0}\right)$ for all $p \in \mathscr{S}^{*}(X), \bigvee_{U \in \mathbf{I}^{X}} \mu(p, U)=1$;

$\left(\mathrm{m}_{1}\right)$ for all $p \in \mathscr{S}^{*}(X)$ and for all $U, V \in \mathbf{I}^{X}$, if $U \leq V$, then $\mu(p, U) \leq \mu(p, V)$;

$\left(\mathrm{m}_{2}\right)$ for all $p \in \mathscr{S}^{*}(X)$ and for all $U_{1}, U_{2} \in \mathbf{I}^{X}, \mu\left(p, U_{1} \wedge U_{2}\right) \geq \mu\left(p, U_{1}\right) \wedge \mu\left(p, U_{2}\right)$;

$\left(\mathrm{m}_{3}\right)$ for all $p \in \mathscr{S}^{*}(X)$ and for all $U \in \mathbf{I}^{X}$, if $\mu(p, U)>0$, then $p \prec U$;

$\left(\mathrm{m}_{4}\right)$ for all $p \in \mathscr{S}^{*}(X)$ and for all $U \in \mathbf{I}^{X}, \mu(p, U) \leq \bigvee_{V \leq U}\left(\mu(p, V) \wedge\left(\bigwedge_{q<V} \mu(q, V)\right)\right)$. We observe that the second member of the inequality in $\left(\mathrm{m}_{4}\right)$, in the presence of the condition $\left(\mathrm{m}_{3}\right)$, shrinks to $\bigvee_{p \prec V \leq U} \bigwedge_{q \prec V} \mu(q, V)$.

Proposition 4.1. Let $\mu \in \mathbf{I}^{\varphi *(X) \times \mathbf{I}^{X}}$.

If $\mu$ satisfies the conditions $\left(\mathrm{m}_{0,1}\right)$, then it also satisfies the condition $\left(\mathrm{m}_{\mathrm{a}}\right)$ for all $p \in \mathscr{S}^{*}(X), \mu(p, X)=1$.

If $\mu$ satisfies the conditions $\left(\mathrm{m}_{1,3,4}\right)$, then it also satisfies the conditions

$\left(\mathrm{m}_{\mathrm{b}}\right)$ for all $p, q \in \mathscr{S}^{*}(X)$ and for all $U \in \mathbf{I}^{X}$, if $q \prec p$, then $\mu(p, U) \leq \mu(q, U)$;

$\left(\mathrm{m}_{\mathrm{m}}\right)$ for all $p \in \mathscr{S}^{*}(X)$ and for all $U \in \mathbf{I}^{X}, \mu(p, U)=\bigvee\left\{\mu(q, U) \mid q \in \mathscr{S}^{*}(X), q \succ p\right\}$.

Proof. The check of $\left(\mathrm{m}_{\mathrm{a}}\right)$ under the conditions $\left(\mathrm{m}_{0,1}\right)$ is immediate.

To prove $\left(\mathrm{m}_{\mathrm{b}}\right)$ under the hypotheses $\left(\mathrm{m}_{1,3,4}\right)$, one can imitate the proof of $\left(\mathrm{k}_{\mathrm{b}}\right)$ in Proposition 3.1.

As for $\left(\mathrm{m}_{\mathrm{m}}\right)$, after acquiring $\left(\mathrm{m}_{\mathrm{b}}\right)$, there remains to prove that if $p \in \mathscr{S}^{*}(X)$ and $U \in$ $\mathbf{I}^{X}$, then $\mu(p, U) \leq \bigvee\left\{\mu(q, U) \mid q \in \mathscr{S}^{*}(X), q \succ p\right\}$, that is, given $\alpha<\mu(p, U)$, there exists $q \in \mathscr{S}^{*}(X)$ such that $q \succ p$ and $\mu(q, U) \geq \alpha$. Due to $\left(\mathrm{m}_{3,4}\right)$, one has $\alpha<\bigvee_{p<V \leq U} \bigwedge_{q<V}$ $\mu(q, V)$, hence there exist $V \in \mathbf{I}^{X}$ such that $p \prec V \leq U$ and $\alpha<\bigwedge_{q<V} \mu(q, V)$. Given any $q \in \mathscr{S}^{*}(X)$ such that $p \prec q \prec V$, we have $\alpha<\mu(q, V)$, so it follows, by $\left(\mathrm{m}_{1}\right)$, that $\alpha<$ $\mu(q, U)$.

With every $\mu \in \mathbf{I}^{\varphi *(X) \times \mathbf{I}^{X}}$ and $\kappa \in \mathbf{I}^{\varphi(X) \times \mathbf{I}^{X}}$, we associate, respectively, the maps $\kappa_{\mu} \in$ $\mathbf{I}^{\varphi(X) \times \mathbf{I}^{X}}$ and $\mu_{\kappa} \in \mathbf{I}^{\varphi *(X) \times \mathbf{I}^{X}}$ defined as follows:

(i) for all $p \in \mathscr{S}(X)$ and for all $U \in \mathbf{I}^{X}, \kappa_{\mu}(p, U)=\bigwedge_{q<p} \mu(q, U)$,

(ii) for all $p \in \mathscr{S}^{*}(X)$ and for all $U \in \mathbf{I}^{X}, \mu_{\kappa}(p, U)=\bigvee_{q \succ p} \kappa(q, U)$.

Example 4.2. Let $X$ be a singleton $\{x\}$. For $\lambda \in(0,1)$ and $U \in \mathbf{I}^{X}$, we define

$$
\mu\left(x_{\lambda}, U\right)= \begin{cases}U(x) & \text { if } \lambda<U(x), \\ 0 & \text { if } \lambda \geq U(x) .\end{cases}
$$

Keeping in mind the function $\kappa$ in Example 3.2, we recognize that $\mu=\mu_{\kappa}$ and $\kappa=\kappa_{\mu}$. 
THEOREM 4.3. For any $\kappa \in \mathbf{I}^{\varphi(X) \times \mathbf{I}^{X}}, \kappa \leq \kappa_{\mu_{\kappa}}$. If $\kappa$ is an $f \cdot k$-prestructure, then $\mu_{\kappa}$ is an $f \cdot m$-structure. If $\kappa$ is an $f \cdot k$-structure, then $\kappa=\kappa_{\mu_{\kappa}}$.

Proof. Given $p \in \mathscr{S}(X)$ and $U \in \mathbf{I}^{X}$, it is evident that for all $q \prec p \kappa(p, U) \leq \bigvee_{r \succ q} \kappa(r, U)=$ $\mu_{\kappa}(q, U)$, so $\kappa(p, U) \leq \bigwedge_{q<p} \mu_{\kappa}(q, U)=\kappa_{\mu_{\kappa}}(p, U)$.

Now we suppose that $\kappa$ is an $f \cdot k$-prestructure. The check of $\left(\mathrm{m}_{0}\right)$ for $\mu_{\kappa}$ is trivial if one considers that $\kappa$ satisfies $\left(\mathrm{k}_{\mathrm{a}}\right)$.

It is immediate to check that if $\kappa$ satisfies $\left(\mathrm{k}_{1}\right)$, then $\mu_{\kappa}$ satisfies $\left(\mathrm{m}_{1}\right)$, and that if $\kappa$ satisfies $\left(\mathrm{k}_{3}\right)$, then $\mu_{\kappa}$ satisfies $\left(\mathrm{m}_{3}\right)$.

To prove $\left(\mathrm{m}_{2}\right)$, let us consider $p \in \mathscr{S}^{*}(X), U_{1}, U_{2} \in \mathbf{I}^{X}, U=U_{1} \wedge U_{2}$, and suppose on the contrary that $\mu_{\kappa}(p, U)<\mu_{\kappa}\left(p, U_{1}\right) \wedge \mu_{\kappa}\left(p, U_{2}\right)$. Then there exist $r, s \succ p$ such that $\mu_{\kappa}(p, U)<\kappa\left(r, U_{1}\right)$ and $\mu_{\kappa}(p, U)<\kappa\left(s, U_{2}\right)$. Thus, assuming, for instance, that $r \preceq s$, one would have, by $\left(\mathrm{k}_{\mathrm{b}}\right)$ and $\left(\mathrm{k}_{2}\right)$, that $\kappa(r, U)<\kappa\left(r, U_{1}\right) \wedge \kappa\left(s, U_{2}\right) \leq \kappa\left(r, U_{1}\right) \wedge \kappa\left(r, U_{2}\right) \leq$ $\kappa(r, U)$.

As for $\left(\mathrm{m}_{4}\right)$, taking $p \in \mathscr{S}^{*}(X), U \in \mathbf{I}^{X}$, and $\alpha<\mu_{\kappa}(p, U)$, we need to prove that there exists $V \in \mathbf{I}^{X}$ such that $p \prec V \leq U$ and $\alpha \leq \bigwedge_{s<V} \mu_{\kappa}(s, V)$. From $\alpha<\bigvee_{q>p} \kappa(q, U)$, it follows that there exists $q \succ p$ such that $\alpha<\kappa(q, U)$. Because of $\left(\mathrm{k}_{3,4}\right)$, one has that there exists $V \in \mathbf{I}^{X}$ such that $p \prec V \leq U$ and $\alpha<\kappa(r, V)$ for all $r \preceq V$. There remains to check that $\alpha \leq \bigwedge_{s<V} \mu_{\kappa}(s, V)$ : in fact, if there existed $s \prec V$ such that $\alpha>\mu_{\kappa}(s, V)$, then, taking $r \in \mathscr{S}(X)$ such that $s \prec r \leq V$, one would have at the same time $\alpha<\kappa(r, V)$ and $\alpha>\kappa(r, V)$.

Now we suppose that $\kappa$ is an $f \cdot k$-structure and we prove that given $p \in \mathscr{Y}(X)$ and $U \in \mathbf{I}^{X}$, it follows that $\kappa(p, U) \geq \kappa_{\mu_{\kappa}}(p, U)$. Since $\kappa_{\mu_{\kappa}}(p, U)=\bigwedge_{q<p} \mu_{\kappa}(q, U)$, the purpose is reached if given $\alpha>\kappa(p, U)$, one finds $q \prec p$ such that $\alpha \geq \mu_{\kappa}(q, U)$. Actually, if $\alpha>$ $\kappa(p, U)$, due to $\left(\mathrm{k}_{5}\right)$ there exists $q \prec p$ such that $\alpha>\kappa(q, U)$, so, by $\left(\mathrm{k}_{\mathrm{b}}\right)$, one has that $\alpha>\kappa(r, U)$ for all $r \succ q$, that is, $\alpha \geq \bigvee_{r \succ q} \kappa(r, U)=\mu_{\kappa}(q, U)$.

Theorem 4.4. For any $\mu \in \mathbf{I}^{\varphi *(X) \times \mathbf{I}^{X}}, \mu \geq \mu_{\kappa_{\mu}}$. If $\mu$ is an $f \cdot m$-structure, then $\kappa_{\mu}$ is an $f \cdot k$ structure and $\mu=\mu_{\kappa_{\mu}}$.

Proof. If $p \in \mathscr{S}^{*}(X)$ and $U \in \mathbf{I}^{X}$, one sees that for all $q \succ p, \mu(p, U) \geq \bigwedge_{r \prec q} \mu(r, U)=$ $\kappa_{\mu}(q, U)$, and hence $\mu(p, U) \geq \bigvee_{q \succ p} \kappa_{\mu}(q, U)=\mu_{\kappa_{\mu}}(p, U)$.

Now let $\mu$ be an $f \cdot m$-structure. The check of the condition $\left(\mathrm{k}_{0}\right)$ for $\kappa_{\mu}$ is trivial if one considers that $\mu$ satisfies $\left(\mathrm{m}_{\mathrm{a}}\right)$.

It is easy to check that for all $i \in\{1,2,3\}, \kappa_{\mu}$ satisfies $\left(\mathrm{k}_{i}\right)$ if $\mu$ satisfies $\left(\mathrm{m}_{i}\right)$.

As for $\left(\mathrm{k}_{4}\right)$, given $p \in \mathscr{S}(X), U \in \mathbf{I}^{X}$, and $\alpha<\kappa_{\mu}(p, U)$, we need to prove that there exists $V \in \mathbf{I}^{X}$ such that $p \preceq V \leq U$ and $\alpha \leq \bigwedge_{s \leq V} \kappa_{\mu}(s, V)$. From $\alpha<\bigwedge_{q<p} \mu(q, U)$, for any $q \prec p$, one has that $\alpha<\mu(q, U)$, and hence, by $\left(\mathrm{m}_{3,4}\right)$, that there exists $V_{q} \in \mathbf{I}^{X}$ such that $q \prec V_{q} \leq U$ and $\alpha<\mu\left(r, V_{q}\right)$ for all $r \prec V_{q}$. Letting $V=\bigvee_{q<p} V_{q}$, we have that $p \preceq V \leq U$, and there remains to prove that given $s \preceq V$, we have $\alpha \leq \kappa_{\mu}(s, V)$. Actually, for all $r \prec s$, since $r \prec V$, there exists $q_{r} \prec p$ such that $r \prec V_{q_{r}}$, so, by $\left(\mathrm{m}_{1}\right)$, one has that $\alpha<\mu\left(r, V_{q_{r}}\right) \leq$ $\mu(r, V)$; thus $\alpha \leq \bigwedge_{r<s} \mu(r, V)=\kappa_{\mu}(s, V)$.

Now we prove that $\kappa_{\mu}$ satisfies $\left(\mathrm{k}_{5}\right)$, that is, for fixed $p \in \mathscr{S}(X)$ and $U \in \mathbf{I}^{X}, \kappa_{\mu}(p, U) \geq$ $\bigwedge_{q<p} \kappa_{\mu}(q, U)$. On the contrary, we suppose that there exists $r \prec p$ such that $\mu(r, U)<$ $\bigwedge_{q<p} \kappa_{\mu}(q, U)$. It follows that, for all $q \prec p, \mu(r, U)<\kappa_{\mu}(q, U)=\bigwedge_{s<q} \mu(s, U)$. Then, taking $q \in \mathscr{S}^{*}(X)$ such that $r \prec q \prec p$, it would follow that $\mu(r, U)<\bigwedge_{s<q} \mu(s, U) \leq \mu(r, U)$. 
Finally, we check that given $p \in \mathscr{S}^{*}(X)$ and $U \in \mathbf{I}^{X}$, we have $\mu(p, U) \leq \mu_{\kappa_{\mu}}(p, U)$. Since $\mu_{\kappa_{\mu}}(p, U)=\bigvee_{q \succ p} \kappa_{\mu}(q, U)$, the purpose is reached if taking $\alpha<\mu(p, U)$, one finds $q \succ p$ such that $\alpha \leq \kappa_{\mu}(q, U)$. Indeed, if $\alpha<\mu(p, U)$, because of $\left(\mathrm{m}_{\mathrm{m}}\right)$ there exists $q \succ p$ such that $\alpha<\mu(q, U)$, so, by $\left(\mathrm{m}_{\mathrm{b}}\right)$, it follows that $\alpha<\mu(r, U)$ for all $r \prec q$, that is, $\alpha \leq \bigwedge_{r<q} \mu(r, U)=$ $\kappa_{\mu}(q, U)$.

If we denote by $f \cdot M(X)$ and $f \cdot K(X)$, respectively, the families of all $f \cdot m$-structures and of all $f \cdot k$-structures on $X$, then because of the two theorems above, we can assert that they are equivalent, namely there exists the bijection

$$
h: \mu \in f \cdot M(X) \longrightarrow \kappa_{\mu} \in f \cdot K(X) .
$$

One recognizes that the inverse of $h$ is the map defined by $h^{-1}(\kappa)=\mu_{\kappa}$ for all $\kappa \in f \cdot K(X)$.

Keeping in mind the results of Section 3, with any $f \cdot m$-structure $\mu$, we can associate the $f$-topology $\mathscr{T}_{h(\mu)}$, which is expressed this way:

$$
\forall A \in \mathbf{I}^{X}, \quad \mathscr{T}_{h(\mu)}(A)=\bigwedge_{p \prec A} \mu(p, A)
$$

By comparing this expression with the one for $\mathscr{T}_{\kappa}(A)$ in the previous section, it seems justified to indicate the $f$-topology $\mathscr{T}_{h(\mu)}$ by the symbol $\mathscr{T}_{\mu}$.

Conversely, with any $f$-topology $\tau$, one can associate the $f \cdot m$-structure $h^{-1}\left(\mathscr{K}_{\tau}\right)$ that we will indicate by the new symbol $M_{\tau}$ in the sequel, which is expressed as follows:

$$
\forall p \in \mathscr{S}^{*}(X), \forall U \in \mathbf{I}^{X}, \quad M_{\tau}(p, U)=\bigvee_{p<A \leq U} \tau(A)
$$

We observe that $\mathscr{T}_{\mu}$ is the only $f$-topology such that $\mu=M_{\mathscr{T}_{\mu}}$ and $M_{\tau}$ is the only $f \cdot m$ structure such that $\tau=\mathscr{T}_{\mu_{\tau}}$.

Theorem 4.5. Let $(X, \tau)$ and $(Y, \delta)$ be two f-topological spaces. The map $f: X \rightarrow Y$ is continuous if and only if for all $p \in \mathscr{S}^{*}(X)$ and for all $V \in \mathbf{I}^{Y}, M_{\tau}\left(p, f^{\leftarrow}(V)\right) \geq M_{\delta}\left(f^{-}(p), V\right)$.

Proof. The proof is straightforward.

If $\mu \in \mathbf{2}^{\varphi^{*}(X) \times \mathbf{I}^{X}}$, by introducing $\mu\langle p\rangle=\left\{U \in \mathbf{I}^{X} \mid \mu(p, U)=1\right\}$ for all $p \in \mathscr{S}^{*}(X)$, we can give to the conditions $\left(\mathrm{m}_{0,1,2,3,4, \mathrm{a}, \mathrm{b}, \mathrm{m}}\right)$ a look similar to the one given to $\left(\mathrm{k}_{0,1,2,3,4, \mathrm{a}, \mathrm{b}, \mathrm{k}}\right)$ in the section above for $\kappa \in 2^{\varphi(X) \times I^{X}}$ (cf. $\left.[2,5]\right)$. In this case, we speak of a $c \cdot$ m-neighborhood structure, or a $c \cdot m$-structure for short. We note that the condition $\left(\mathrm{m}_{\mathrm{m}}\right)$ becomes the following:

$\left(\mathrm{m}_{\mathrm{m}}^{c}\right)$ for all $p \in \mathscr{S}^{*}(X), \kappa\langle p\rangle=\bigcup\left\{\mu\langle q\rangle \mid q \in \mathscr{S}^{*}(X)\right.$ and $\left.q>p\right\}$, which is a particular case of that property of the $c \cdot m$-structure which in [8, Proposition $2]$ is called the "supcompleteness property."

The results stated in this section allow us to assert that if $\tau$ is a $c$-topology, there remains associated with it the $c \cdot m$-structure $M_{\tau}$ such that for all $p \in \mathscr{S}^{*}(X), M_{\tau}\langle p\rangle=$ $\left\{U \in \mathbf{I}^{X} \mid \exists A \in \tau\right.$ such that $\left.p \prec A \leq U\right\}$, which is the only $c \cdot m$-structure such that for 
any $A \in \mathbf{I}^{X}, A \in \tau \Leftrightarrow A \in M_{\tau}\langle p\rangle$ for all $p \prec A$. The elements of $M_{\tau}\langle p\rangle$ are the $m$-neighborhoods of $p$ with respect to $\tau$. If conversely $\mu$ is a $c \cdot m$-structure, there remains associated with it the $c$-topology $\mathscr{T}_{\mu}=\left\{A \in \mathbf{I}^{X} \mid A \in \mu\langle p\rangle \forall p \prec A\right\}$, which is the only $c$-topology such that for any $p \in \mathscr{S}^{*}(X)$ and $U \in \mathbf{I}^{X}, U \in \mu\langle p\rangle \Leftrightarrow \exists A \in \mathscr{T}_{\mu}$ such that $p \prec A \leq U$. All this is in accord with the results on $m$-neighborhoods gathered in [7].

From Theorems 4.3 and 4.4, it follows that with the $c \cdot k$-structure $\kappa$ and the $c$. $m$-structure $\mu$ are associated, respectively, the $c \cdot m$-structure $\mu_{\kappa}$ and the $c \cdot k$-structure $\kappa_{\mu}$ defined this way: for all $p \in \mathscr{S}^{*}(X), \mu_{\kappa}\langle p\rangle=\bigcup_{q\rangle p} \kappa\langle q\rangle$; for all $p \in \mathscr{S}(X), \kappa_{\mu}\langle p\rangle=$ $\bigcap_{q<p} \mu\langle q\rangle$. These equalities agree with the corresponding equalities in [8, Proposition 1], where the case is considered in which $\kappa$ and $\mu$ are, respectively, the $c \cdot k$-structure $\mathcal{K}_{\tau}$ and the $c \cdot m$-structure $M_{\tau}$ associated with the same $c$-topology $\tau$ (in this case, it follows that $\kappa_{\mu}=\kappa$ and $\mu_{\kappa}=\mu$ ).

\section{5. $q$-neighborhood structures}

We call every map $\pi \in \mathbf{I}^{\varphi(X) \times \mathbf{I}^{X}}$ verifying the following axioms an $f \cdot q$-neighborhood structure, or an $f \cdot q$-structure, on the nonempty set $X$, (see $[3,11])$ :

$\left(\mathrm{q}_{0}\right)$ for all $p \in \mathscr{S}(X), \bigvee_{U \in \mathrm{I}^{X}} \pi(p, U)=1$;

$\left(\mathrm{q}_{1}\right)$ for all $p \in \mathscr{S}(X)$ and for all $U, V \in \mathbf{I}^{X}$, if $U \leq V$, then $\pi(p, U) \leq \pi(p, V)$;

$\left(\mathrm{q}_{2}\right)$ for all $p \in \mathscr{S}(X)$ and for all $U_{1}, U_{2} \in \mathbf{I}^{X}, \pi\left(p, U_{1} \wedge U_{2}\right) \geq \pi\left(p, U_{1}\right) \wedge \pi\left(p, U_{2}\right)$;

( $\left.\mathrm{q}_{3}\right)$ for all $p \in \mathscr{S}(X)$ and for all $U \in \mathbf{I}^{X}$, if $\pi(p, U)>0$, then $p \hat{\mathrm{q}} U$;

$\left(\mathrm{q}_{4}\right)$ for all $p \in \mathscr{S}(X)$ and for all $U \in \mathbf{I}^{X}, \pi(p, U) \leq \bigvee_{V \leq U}\left(\pi(p, V) \wedge\left(\bigwedge_{q \hat{\mathrm{q}} V} \pi(q, V)\right)\right)$. We observe that the second member of the inequality in $\left(\mathrm{q}_{4}\right)$, in the presence of the condition ( $\left.\mathrm{q}_{3}\right)$, shrinks to $\bigvee_{p \hat{\mathrm{q}} V \leq U} \bigwedge_{q \hat{\mathrm{q}} V} \pi(q, V)$.

It is easy to check that the notion of $f \cdot q$-structure is equivalent to the one of " $I$-fuzzy quasi-coincident neighborhood system" [4].

Proposition 5.1. Let $\pi \in \mathbf{I}^{\varphi(X) \times \mathbf{I}^{X}}$. If $\pi$ satisfies the conditions $\left(\mathrm{q}_{0,1}\right)$, then it also satisfies the condition

( $\left.\mathrm{q}_{\mathrm{a}}\right)$ for all $p \in \mathscr{S}(X), \pi(p, X)=1$.

If $\pi$ satisfies the conditions ( $\left.\mathrm{q}_{1,3,4}\right)$, then it also satisfies the following conditions:

( $\mathrm{q}_{\mathrm{b}}$ ) for all $p, q \in \mathscr{S}(X)$ and for all $U \in \mathbf{I}^{X}$, if $q \succ p$, then $\pi(p, U) \leq \pi(q, U)$;

( $\mathrm{q}_{\mathrm{q}}$ ) for all $p \in \mathscr{S}(X)$ and for all $U \in \mathbf{I}^{X}, \pi(p, U)=\bigvee_{q<p} \pi(q, U)$.

Proof. The check of $\left(\mathrm{q}_{\mathrm{a}}\right)$ under the conditions $\left(\mathrm{q}_{0,1}\right)$ is immediate.

To prove $\left(\mathrm{q}_{\mathrm{b}}\right)$ under the hypothesis $\left(\mathrm{q}_{1,3,4}\right)$, one can imitate the proof of $\left(\mathrm{k}_{\mathrm{b}}\right)$ in Proposition 3.1 (at one point, one will have to observe that from $p \hat{\mathrm{q}} V$ and $q \succ p$, it follows that $q \hat{\mathrm{q}} V)$.

As for $\left(\mathrm{q}_{\mathrm{q}}\right)$, after acquiring $\left(\mathrm{q}_{\mathrm{b}}\right)$, there remains to prove that given $p \in \mathscr{S}(X)$ and $U \in \mathbf{I}^{X}$, it follows that $\pi(p, U) \leq \bigvee_{q<p} \pi(q, U)$, that is, given $\alpha<\pi(p, U)$, there exists $q \prec p$ such that $\pi(q, U) \geq \alpha$. Due to $\left(\mathrm{q}_{3,4}\right)$, it holds that $\alpha<\bigvee_{p \hat{\mathrm{q}} V \leq U} \bigwedge\{\pi(q, V) \mid q \in$ $\mathscr{S}^{*}(X)$ and $\left.q \hat{\mathrm{q}} V\right\}$, and hence there exists $V \in \mathbf{I}^{X}$ such that $p \hat{\mathrm{q}} V \leq U$ and $\alpha<\bigwedge\{\pi(q, V) \mid$ $\mid q \in \mathscr{S}^{*}(X)$ and $\left.q \hat{\mathrm{q}} V\right\}$. Given any $q \prec p$ such that $q \hat{\mathrm{q}} V$, one has $\alpha<\pi(q, V)$, so it turns out, by $\left(\mathrm{q}_{1}\right)$, that $\alpha<\pi(q, U)$. 
Let us denote by $f \cdot Q(X)$ the family of all $f \cdot q$-structures on $X$ and consider the map $l$ defined as follows:

$$
\forall \pi \in f \cdot Q(X), \quad l(\pi)(p, U)=\pi\left(p^{\prime}, U\right) \quad \forall p \in \mathscr{S}^{*}(X), \forall U \in \mathbf{I}^{X}
$$

The theorem below states that $l$ is bijective between the families $f \cdot Q(X)$ and $f \cdot M(X)$, and that the inverse of $l$ is expressed this way: for all $\mu \in f \cdot M(X), l^{-1}(\mu)(p, U)=$ $\bigvee\left\{\mu(q, U) \mid q \in \mathscr{S}^{*}(X)\right.$ and $\left.q \hat{\mathrm{q}} p\right\}$ for all $p \in \mathscr{S}^{*}(X)$ and for all $U \in \mathbf{I}^{X}$. One recognizes, using $\left(\mathrm{m}_{\mathrm{m}}\right)$, that if $p \in \mathscr{S}^{*}(X)$, then $l^{-1}(\mu)(p, U)=\mu\left(p^{\prime}, U\right)$; if instead $p \notin \mathscr{S}^{*}(X)$, then $l^{-1}(\mu)(p, U)=\bigvee_{q<p} \mu(q, U)$. In the sequel, we will write also $\mu_{\pi}$ in place of $l(\pi)$ and $\pi_{\mu}$ in place of $l^{-1}(\mu)$.

Example 5.2. Let $X$ be a singleton $\{x\}$. For $\lambda \in(0,1]$ and $U \in \mathbf{I}^{X}$, we define

$$
\pi\left(x_{\lambda}, U\right)= \begin{cases}0 & \text { if } \lambda \leq 1-U(x) \\ U(x) & \text { if } \lambda>1-U(x) .\end{cases}
$$

Keeping in mind the function $\mu$ in Example 4.2, we recognize that $\pi=\pi_{\mu}$ and $\mu=\mu_{\pi}$.

Theorem 5.3. The map $l$ introduced above is bijective between $f \cdot Q(X)$ and $f \cdot M(X)$. Its inverse $l^{-1}$ is the map defined as follows: for all $\mu \in f \cdot M(X), l^{-1}(\mu)(p, U)=\bigvee\{\mu(q, U) \mid$ $q \in \mathscr{S}^{*}(X)$ and $\left.q \hat{\mathrm{q}} p\right\}$ for all $p \in \mathscr{S}^{*}(X)$ and for all $U \in \mathbf{I}^{X}$.

Proof. It is easy to check that if $\pi \in f \cdot Q(X)$, since $\pi$ satisfies $\left(\mathrm{q}_{i}\right)$, for each $i \in\{0,1,2,3,4\}$, then $\mu_{\pi}$ satisfies $\left(\mathrm{m}_{i}\right)$ (in particular, with regard to $\left(\mathrm{m}_{4}\right)$, it is useful to observe that, for all $V \in \mathbf{I}^{X}$, it follows that $\bigwedge_{q \hat{q} V} \pi(q, V) \leq \bigwedge\left\{\pi(q, V) \mid q \in \mathscr{S}^{*}(X)\right.$ and $\left.q \hat{\mathrm{q}} V\right\}=\bigwedge_{q<V} \pi\left(q^{\prime}\right.$, $\left.V)=\bigwedge_{q<V} \mu_{\pi}(q, V)\right)$.

To prove that $l$ is injective, given $\pi, \sigma \in f \cdot Q(X)$ such that $l(\pi)=l(\sigma)$ and fixed $p \in$ $\mathscr{S}(X)$ and $U \in \mathbf{I}^{X}$, we check that $\pi(p, U)=\sigma(p, U)$. If $p \in \mathscr{S}^{*}(X)$, we have $\pi(p, U)=$ $\mu_{\pi}\left(p^{\prime}, U\right)=\mu_{\sigma}\left(p^{\prime}, U\right)=\sigma(p, U)$; if $p \notin \mathscr{S}^{*}(X)$, using $\left(\mathrm{q}_{\mathrm{q}}\right)$, one has that $\pi(p, U)=\bigvee_{q<p} \pi$ $(q, U)=\bigvee_{q<p} \mu_{\pi}\left(q^{\prime}, U\right)=\bigvee_{q<p} \mu_{\sigma}\left(q^{\prime}, U\right)=\bigvee_{q<p} \sigma(q, U)=\sigma(p, U)$.

To complete the proof, we take $\mu \in f \cdot M(X)$, consider the map $\pi_{\mu}$ defined by $\pi_{\mu}$ $(p, U)=\bigvee\left\{\mu(q, U) \mid q \in \mathscr{S}^{*}(X)\right.$ and $\left.q \hat{\mathrm{q}} p\right\}$, and check that $\pi_{\mu} \in f \cdot Q(X)$ and $l\left(\pi_{\mu}\right)=\mu$.

One easily verifies that for $i \in\{0,1,3\}$, since $\mu$ satisfies $\left(\mathrm{m}_{i}\right)$, then $\pi_{\mu}$ satisfies $\left(\mathrm{q}_{i}\right)$. Now we prove that since $\mu$ satisfies $\left(\mathrm{m}_{1,2,3,4}\right)$, then $\pi_{\mu}$ satisfies $\left(\mathrm{q}_{2}\right)$. Considering $p \in \mathscr{S}(X) \backslash$ $\mathscr{S}^{*}(X)$ (the case when $p \in \mathscr{S}^{*}(X)$ would be trivial) and $U_{1}, U_{2} \in \mathbf{I}^{X}$, let $U=U_{1} \wedge U_{2}$ and suppose on the contrary that $\pi_{\mu}(p, U)<\pi_{\mu}\left(p, U_{1}\right) \wedge \pi_{\mu}\left(p, U_{2}\right)$. Now there exist $r, s \prec$ $p$ such that $\pi_{\mu}(p, U)<\mu\left(r, U_{1}\right)$ and $\pi_{\mu}(p, U)<\mu\left(s, U_{2}\right)$. Then, assuming, for instance, $r \preceq s$, one would have, by $\left(\mathrm{m}_{\mathrm{b}}\right)$ and $\left(\mathrm{m}_{2}\right)$, that $\pi_{\mu}(p, U)<\mu\left(r, U_{1}\right) \wedge \mu\left(s, U_{2}\right) \leq \mu\left(r, U_{1}\right) \wedge$ $\mu\left(r, U_{2}\right) \leq \mu(r, U)$. Now we prove that since $\mu$ satisfies $\left(\mathrm{m}_{1,3,4}\right)$, then $\pi_{\mu}$ satisfies $\left(\mathrm{q}_{4}\right)$. So let us firstly observe that given $V \in \mathbf{I}^{X}$, one has that $\bigwedge_{r<V} \mu(r, V) \leq \bigwedge_{q} \hat{\mathrm{q}} V \pi_{\mu}(q, V)$; indeed, if $q \hat{\mathrm{q}} V$ existed such that $\pi_{\mu}(q, V)<\bigwedge_{r<V} \mu(r, V)$, then given $r, s \in \mathscr{S}^{*}(X)$ such that $s \hat{\mathrm{q}} q$ and $s \prec r \prec V$, we would obtain $\mu(s, V) \leq \pi_{\mu}(q, V)<\mu(r, V)$, in contrast with $\left(\mathrm{m}_{\mathrm{b}}\right)$. Hence, given $p \in \mathscr{S}(X)$ and $U \in \mathbf{I}^{X}$, one sees that $\pi_{\mu}(p, U)=\bigvee\left\{\mu(q, U) \mid q \in \mathscr{S}^{*}(X)\right.$ and $\left.q \hat{\mathrm{q}} p\right\} \leq$ $\bigvee\left\{\bigvee_{q<V \leq U} \bigwedge_{r<V} \mu(r, V) \mid q \in \mathscr{S}^{*}(X)\right.$ and $\left.q \hat{\mathrm{q}} p\right\}=\bigvee_{p \hat{\mathrm{q}} V \leq U} \bigwedge_{r<V} \mu(r, V) \leq \bigvee_{p \hat{\mathrm{q}} V \leq U}$ $\bigwedge_{q \hat{\mathrm{q}} V} \pi_{\mu}(q, V)$. 
At last, for any $p \in \mathscr{S}^{*}(X)$ and $U \in \mathbf{I}^{X}$, we compute that $l\left(\pi_{\mu}\right)(p, U)=\pi_{\mu}\left(p^{\prime}, U\right)=$ $\mu(p, U)$.

Keeping in mind the results of Section 4 , with any $f \cdot q$-structure $\pi$ we can associate the $f$-topology $\mathscr{T}_{l(\pi)}$, denoted by $\mathscr{E}_{\pi}$ in the sequel, which is defined by

$$
\forall A \in \mathbf{I}^{X}, \quad \mathscr{E}_{\pi}(A)=\bigwedge_{p \widehat{\mathrm{q}} A} \pi(p, A) .
$$

In fact, for all $A \in \mathbf{I}^{X}, \mathscr{E}_{\pi}(A)=\bigwedge_{p \prec A} \mu_{\pi}(p, A)=\bigwedge_{p \prec A} \pi\left(p^{\prime}, A\right)=\bigwedge\left\{\pi(p, A) \mid p \in \mathscr{S}^{*}(X)\right.$ and $p \hat{\mathrm{q}} A\}=\bigwedge_{p \hat{\mathrm{q}} A} \pi(p, A)$, where, to explain the nontrivial inequality $\bigwedge\{\pi(p, A) \mid p$ $\in \mathscr{S}^{*}(X)$ and $\left.p \hat{\mathrm{q}} A\right\} \leq \bigwedge_{p \hat{\mathrm{q}} A} \pi(p, A)$, we note that given $q \in \mathscr{Y}(X)$ such that $q \hat{\mathrm{q}} A$, if we had $\pi(q, A)<\bigwedge\{\pi(p, A) \mid p \in \mathscr{S} *(X)$ and $p \hat{\mathrm{q}} A\}$, then it would follow that $\pi(q, A)<$ $\pi(p, A)$, which contradicts ( $\left.\mathrm{q}_{\mathrm{b}}\right)$ if $p \prec q$.

Conversely, with any $f$-topology $\tau$, one can associate the $f \cdot q$-structure $l^{-1}\left(M_{\tau}\right)$, denoted by $\mathscr{2}_{\tau}$ in the sequel, which is expressed as follows:

$$
\forall p \in \mathscr{T}(X), \forall U \in \mathbf{I}^{X}, \quad \mathscr{2}_{\tau}(p, U)=\bigvee_{p \hat{\mathrm{q}} A \leq U} \tau(A)
$$

In fact, for all $p \in \mathscr{S}(X)$ and for all $U \in \mathbf{I}^{X}, \mathscr{Q}_{\tau}(p, U)=\pi_{\mu_{\tau}}(p, U)=\bigvee\left\{M_{\tau}(q, U) \mid q \in\right.$ $\mathscr{S}^{*}(X)$ and $\left.q \hat{\mathrm{q}} p\right\}=\bigvee\left\{\bigvee_{q<A \leq U} \tau(A) \mid q \in \mathscr{S}^{*}(X)\right.$ and $\left.q \hat{\mathrm{q}} p\right\}=\bigvee_{p \hat{\mathrm{q}} A \leq U} \tau(A)$.

We observe that $\mathscr{E}_{\pi}$ is the only $f$-topology such that $\pi=\mathscr{2}_{\mathscr{E}_{\pi}}$ and $\mathscr{Q}_{\tau}$ is the only $f \cdot q$ structure such that $\tau=\mathscr{E}_{2}$.

All this agrees with the results on $q$-neighborhoods stated in $[3,4,11]$.

Theorem 5.4. Let $(X, \tau)$ and $(Y, \delta)$ be two f-topological spaces. The map $f: X \rightarrow Y$ is continuous if and only if for all $p \in \mathscr{S}(X)$ and for all $V \in \mathbf{I}^{Y}, \mathscr{2}_{\tau}\left(p, f^{-}(V)\right) \geq \mathscr{2}_{\delta}\left(f^{\rightarrow}(p), V\right)$.

For the proof see [4, Propositions 3.6 and 3.7].

If $\pi \in 2^{\mathscr{S}(X) \times \mathbf{I}^{X}}$, by introducing $\pi\langle p\rangle=\left\{U \in \mathbf{I}^{X} \mid \pi(p, U)=1\right\}$ for all $p \in \mathscr{S}(X)$, we can give to the conditions $\left(\mathrm{q}_{0,1,2,3,4, \mathrm{a}, \mathrm{b}, \mathrm{q}}\right)$ a look similar to the one given to $\left(\mathrm{k}_{0,1,2,3,4, \mathrm{a}, \mathrm{b}, \mathrm{k}}\right)$ in Section 3 for $\kappa \in 2^{\varphi(X) \times \mathbf{I}^{X}}$ (the conditions $\left(\mathrm{q}_{1,2,3,4}^{c}\right)$ so obtained are the same as in [9]). In this case, we speak of $c \cdot q$-neighborhood structure, or $c \cdot q$-structure for short. We note that the condition $\left(\mathrm{q}_{\mathrm{q}}\right)$ becomes the following:

$\left(\mathrm{q}_{\mathrm{q}}^{c}\right)$ for all $p \in \mathscr{S}(X), \pi\langle p\rangle=\bigcup_{q<p} \pi\langle q\rangle$,

which is a particular case of that property of the $c \cdot q$-structures which in [8, Proposition $2]$ is called the "sup-completeness property."

The results achieved in this section allow us to assert that if $\tau$ is a $c$-topology, there remains associated with it the $c \cdot q$-structure $\mathscr{2}_{\tau}$ given by for all $p \in \mathscr{S}(X), \mathscr{2}_{\tau}\langle p\rangle=\{U \in$ $\mathbf{I}^{X} \mid \exists A \in \tau$ such that $\left.p \hat{\mathrm{q}} A \leq U\right\}$, which is the only $c \cdot q$-structure such that for any $A \in$ $\mathbf{I}^{X}, A \in \tau \Leftrightarrow A \in \mathscr{2}_{\tau}\langle p\rangle$ for all $p \hat{\mathrm{q}} A$. The elements of $\mathscr{2}_{\tau}\langle p\rangle$ are the $q$-neighborhoods of $p$ with respect to $\tau$. If conversely $\pi$ is a $c \cdot q$-structure, there remains associated with it the $c$-topology $\mathscr{E}_{\pi}=\left\{A \in \mathbf{I}^{X} \mid A \in \pi\langle p\rangle \forall p \hat{\mathrm{q}} A\right\}$, which is the only $c$-topology such that for any $p \in \mathscr{Y}(X)$ and $U \in \mathbf{I}^{X}, U \in \pi\langle p\rangle \Leftrightarrow \exists A \in \mathscr{E}_{\pi}$ such that $p \hat{\mathrm{q}} A \leq U$. All this is in accordance with the results on the $q$-neighborhoods gathered in [7]. 
From Theorem 4.3, we see that with the $c \cdot m$-structure $\mu$ and the $c \cdot q$-structure $\pi$ are associated, respectively, the $c \cdot q$-structure $\pi_{\mu}$ and the $c \cdot m$-structure $\mu_{\pi}$ defined as follows: for all $p \in \mathscr{Y}(X), \pi_{\mu}\langle p\rangle=\bigcup\left\{\mu\langle q\rangle \mid q \in \mathscr{S}^{*}(X)\right.$ and $\left.q \hat{\mathrm{q}} p\right\}$, that is, $\pi_{\mu}\langle p\rangle=\mu\left\langle p^{\prime}\right\rangle$ if $p \in \mathscr{S}^{*}(X)$ and $\pi_{\mu}\langle p\rangle=\bigcup_{q<p} \mu\langle q\rangle$ if $p \notin \mathscr{S} *(X)$; for all $p \in \mathscr{S}^{*}(X), \mu_{\pi}\langle p\rangle=\pi\left\langle p^{\prime}\right\rangle$. These equalities agree with the corresponding equalities in [8, Proposition 1$]$, where the case is considered in which $\mu$ and $\pi$ are, respectively, the $c \cdot m$-structure $\mu_{\tau}$ and the $c \cdot q$ structure $2_{\tau}$ associated with the same $c$-topology $\tau$ (in this case, we have $\mu_{\pi}=\mu$ and $\left.\pi_{\mu}=\pi\right)$.

\section{References}

[1] C. De Mitri and E. Pascali, Characterization of fuzzy topologies from neighborhoods of fuzzy points, J. Math. Anal. Appl. 93 (1983), no. 1, 1-14.

[2] M. Á. de Prada Vicente, Neighborhoods of fuzzy points and continuity, Proc. 8th PortugueseSpanish Conference on Mathematics, Vol. I (Coimbra, 1981), Univ. Coimbra, Coimbra, 1981, pp. 357-362.

[3] M. Demirci, Neighborhood structures of smooth topological spaces, Fuzzy Sets and Systems 92 (1997), no. 1, 123-128.

[4] J. Fang, I-FTOP is isomorphic to I-FQN and I-AITOP, Fuzzy Sets and Systems 147 (2004), no. 2, 317-325.

[5] M. H. Ghanim and A. S. Mashhour, Characterization of fuzzy topologies by s-neighborhood systems, Fuzzy Sets and Systems 9 (1983), no. 2, 211-213.

[6] E. E. Kerre, Fuzzy Topologizing With Preassigned Operations, International Congress for Mathematicians, Helsinki, 1978.

[7] E. E. Kerre and P. L. Ottoy, On the different notions of neighbourhood in Chang-Goguen fuzzy topological spaces, Simon Stevin 61 (1987), no. 2, 131-146.

[8] L L L Lattice properties of neighbourhood systems in Chang fuzzy topological spaces, Fuzzy Sets and Systems 30 (1989), no. 2, 205-213.

[9] P. M. Pu and Y. M. Liu, Fuzzy topology. I. Neighborhood structure of a fuzzy point and MooreSmith convergence, J. Math. Anal. Appl. 76 (1980), no. 2, 571-599.

[10] A. P. Šostak, On a fuzzy topological structure, Rend. Circ. Mat. Palermo (2) Suppl. (1985), no. 11, 89-103 (1987).

[11] On the neighborhood structure of fuzzy topological spaces, Zb. Rad. (1990), no. 4, 7-14.

[12] M. S. Ying, A new approach for fuzzy topology. I, Fuzzy Sets and Systems 39 (1991), no. 3, 303321.

[13] On the method of neighborhood systems in fuzzy topology, Fuzzy Sets and Systems 68 (1994), no. 2, 227-238.

Cosimo De Mitri: Department of Mathematics "Ennio De Giorgi”, University of Lecce, via Arnesano, C.P. 193, 73100 Lecce, Italy

E-mail address: cosimo.demitri@unile.it 


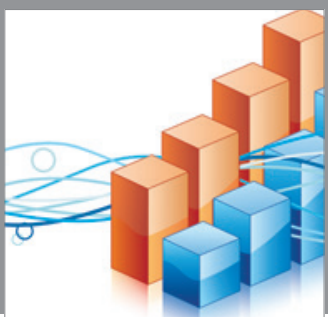

Advances in

Operations Research

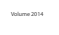

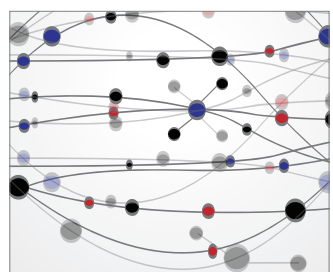

\section{The Scientific} World Journal
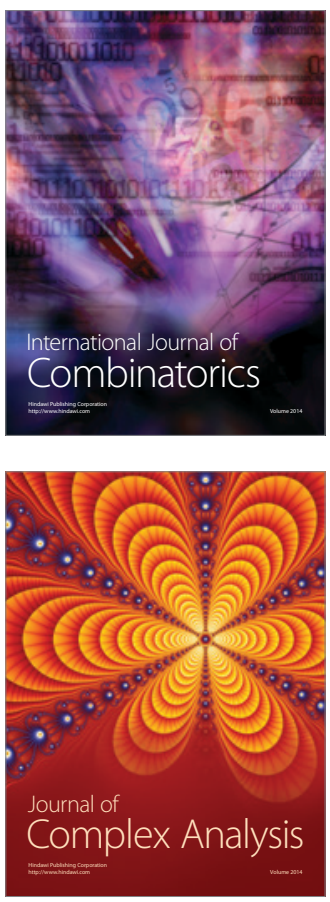

International Journal of

Mathematics and

Mathematical

Sciences
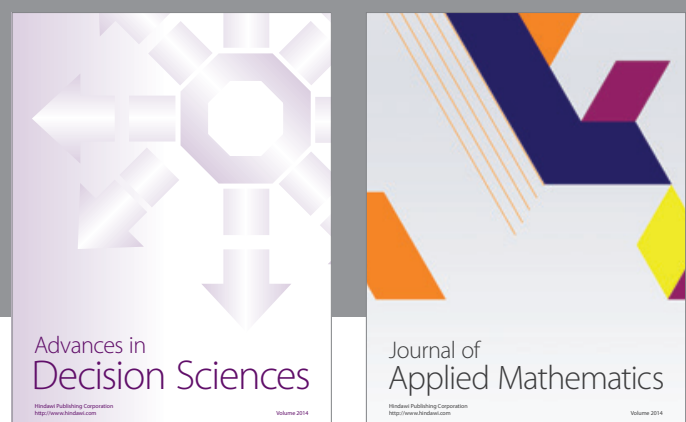

Journal of

Applied Mathematics
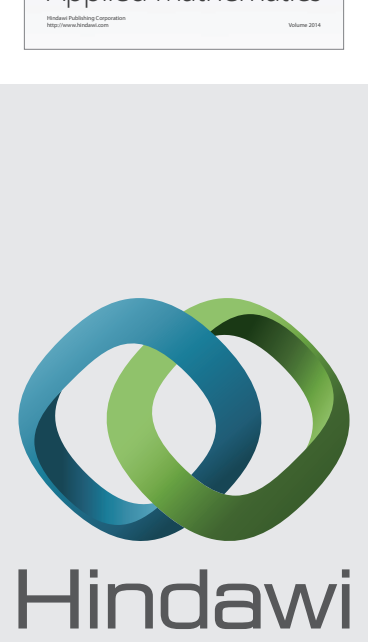

Submit your manuscripts at http://www.hindawi.com
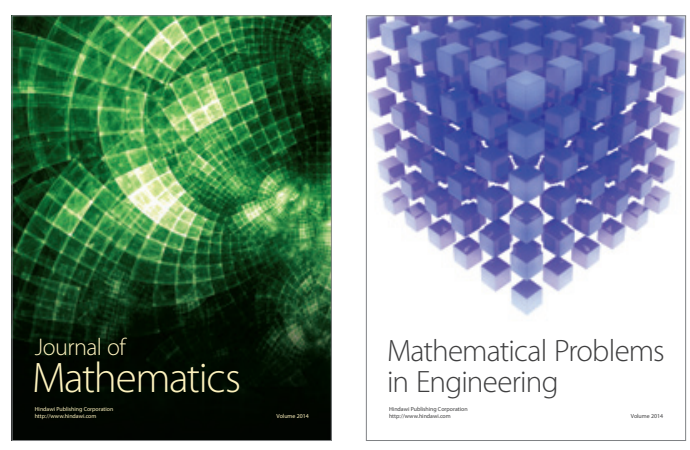

Mathematical Problems in Engineering
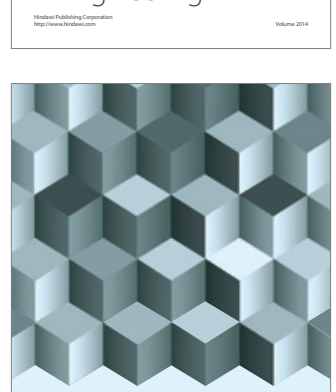

Journal of

Function Spaces
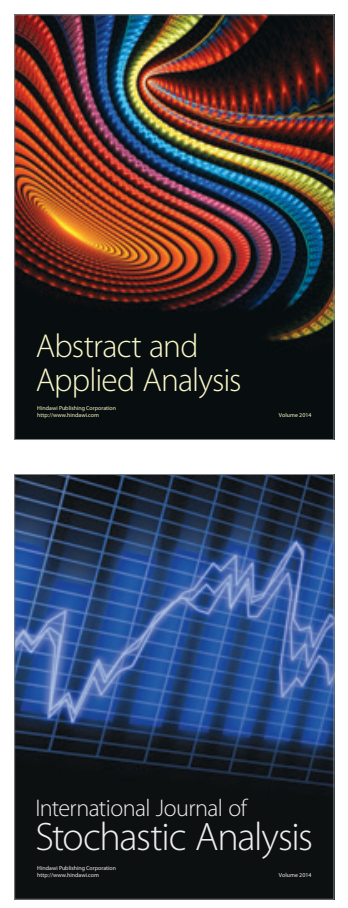

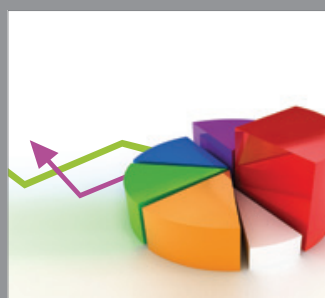

ournal of

Probability and Statistics

Promensencen
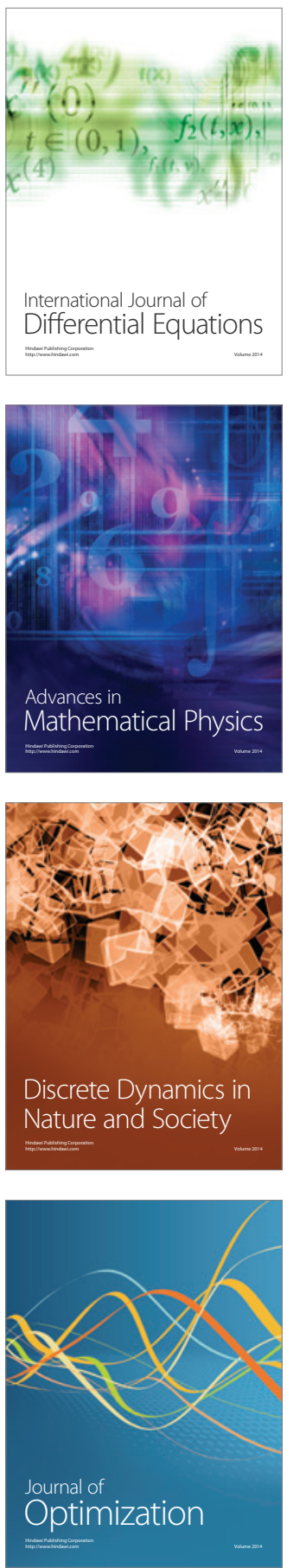